\title{
Pigment Dispersing Factor-Dependent and -Independent Circadian Locomotor Behavioral Rhythms
}

\author{
Vasu Sheeba, ${ }^{1}$ Vijay K. Sharma, ${ }^{1,3}$ Huaiyu Gu, ${ }^{2}$ Yu-Ting Chou, ${ }^{1}$ Diane K. O’Dowd, ${ }^{2}$ and Todd C. Holmes ${ }^{1}$ \\ Departments of ${ }^{1}$ Physiology and Biophysics, and ${ }^{2}$ Developmental and Cell Biology, Anatomy, and Neurobiology, University of California, Irvine, California \\ 92612, and ${ }^{3}$ Evolutionary and Organismal Biology Unit, Jawaharlal Nehru Centre for Advanced Scientific Research, 560064 Bangalore, India
}

Circadian pacemaker circuits consist of ensembles of neurons, each expressing molecular oscillations, but how circuit-wide coordination of multiple oscillators regulates rhythmic physiological and behavioral outputs remains an open question. To investigate the relationship between the pattern of oscillator phase throughout the circadian pacemaker circuit and locomotor activity rhythms in Drosophila, we perturbed the electrical activity and pigment dispersing factor (PDF) levels of the lateral ventral neurons (LNv) and assayed their combinatorial effect on molecular oscillations in different parts of the circuit and on locomotor activity behavior. Altered electrical activity of PDF-expressing LNv causes initial behavioral arrhythmicity followed by gradual long-term emergence of two concurrent short- and long-period circadian behavioral activity bouts in $\sim 60 \%$ of flies. Initial desynchrony of circuit-wide molecular oscillations is followed by the emergence of a novel pattern of period (PER) synchrony whereby two subgroups of dorsal neurons (DN1 and DN2) exhibit PER oscillation peaks coinciding with two activity bouts, whereas other neuronal subgroups exhibit a single PER peak coinciding with one of the two activity bouts. The emergence of this novel pattern of circuit-wide oscillator synchrony is not accompanied by concurrent change in the electrical activity of the LNv. In PDF-null flies, altered electrical activity of LNv drives a short-period circadian activity bout only, indicating that PDF-independent factors underlie the short-period circadian activity component and that the long-period circadian component is PDF-dependent. Thus, polyrhythmic behavioral patterns in electrically manipulated flies are regulated by circuit-wide coordination of molecular oscillations and electrical activity of LNv via PDF-dependent and -independent factors.

Key words: circadian; neural-network; Drosophila; synchronization; peptide modulation; voltage clamp

\section{Introduction}

When Drosophila melanogaster are subjected to periodic light/ dark regimens, the activity/rest rhythm is characterized by two peaks: one around dawn and another around dusk. In the absence of environmental time cues, such as in constant darkness (DD), wild-type flies exhibit a near $24 \mathrm{~h}$ (circadian) rhythm in activity and rest, but gradually lose the morning peak and exhibit a single peak corresponding to evening activity. The underlying circadian pacemaker circuit consists of at least six neuronal subgroups (for review, see Helfrich-Förster et al., 2007). The laterally located neuronal groups (LN) include the large (lLNv) and small ventral lateral neurons and (sLNv) and a dorsal lateral group (LNd). In the dorsal region, are three subgroups of dorsal neurons (DN 1, 2 and 3). The LNv express a peptide neurotransmitter pigment dispersing factor (PDF) with the exception of one of five sLNv henceforth referred to as the fifth sLNv. The level of clock pro-

Received Sept. 6, 2007; revised Nov. 15, 2007; accepted Nov. 20, 2007.

This work was supported by National Science Foundation Grants IBN-0323466 and IBN-0092753, National Institutes of Health (NIH) Grants NS046750 and DA016352 (T.C.H.), and NIH Grant NS27501 (D.K.O.D.). We thank Benjamin White, Michael Nitabach, Jay Park, Paul Taghert, Ralf Stanewsky, and Jeff Hall for sharing reagents; Bálint Rubovszky for technical assistance on electrophysiology experiments; Rachel Wilson for advice on cell-fills; Claude Desplan, Gina Turrigiano, Maki Kaneko, Jeff Hall, and two anonymous reviewers for constructive comments on previous versions of this manuscript; and the Bloomington Stock Center for fly lines.

Correspondence should be addressed to Todd C. Holmes, Department of Physiology and Biophysics, University of California, Irvine, CA 92612. E-mail: tholmes@uci.edu.

DOI:10.1523/JNEUROSCI.4087-07.2008

Copyright $\odot 2008$ Society for Neuroscience $\quad$ 0270-6474/08/280217-11\$15.00/0 teins Period (PER) and Timeless (TIM) oscillate with a daily rhythm, such that highest levels occur around the end of the night or early morning and lowest levels during mid-day (for review, see $\mathrm{Yu}$ and Hardin, 2006). Interestingly this pattern of clock protein oscillation is synchronous in almost all subgroups of pacemaker neurons in the adult fly when entrained by time cues such as light/dark (LD) cycles or temperature cycles (Kaneko et al., 1997; Miyasako et al., 2007). Based on measurements of molecular oscillations in the different subgroups in $p d f^{01}$ null flies (Peng et al., 2003; Lin et al., 2004) and phase shifts in locomotor activity rhythm by peptide injection in the cockroach brain (Petri and Stengl, 1997) it has been postulated that PDF is responsible for the synchrony in molecular oscillations both within and among pacemaker neuronal subgroups. The electrical properties of the pacemaker neurons are critical for normal circadian behavioral and molecular rhythms (Nitabach et al., 2002; Nitabach et al., 2005; Nitabach et al., 2006; de la Paz Fernandez et al., 2007). We have shown previously that targeted expression of a voltage-gated sodium channel ( $\mathrm{NaChBac}$ ) in LNv neurons results in polyrhythmic free-running bouts of locomotor activity under DD that persists for several weeks. Furthermore, the underlying molecular oscillations among the different neuronal subgroups were desynchronized after several days in darkness and such effects were likely to be mediated via PDF (Nitabach et al., 2006). To investigate the apparent anomaly of asynchronous molecular oscillation after short-term exposure to constant darkness while rhythmic 
(albeit, complex) activity persisted under long-term exposure to constant darkness, we conducted a more detailed analysis of the behavioral phenotypes. We also examined the PDF dependence of complex rhythmicity in a $p d f^{\ominus 1}$ null genetic background. Using whole-cell current-clamp recording, we verified that membrane electrical properties of the target neurons are altered as a result of $\mathrm{NaChBac}$ expression and examined whether these properties are altered by compensatory mechanisms in the circuit. We also tested the hypothesis that chronic electrical manipulation results in circuit-level compensatory changes by using the oscillation of clock protein levels as a marker of the phase of the clock in the different components of the neural circuit.

\section{Materials and Methods}

Transgenic lines. Two transgenic fly lines containing the $\mathrm{NaChBac}$ ion channel transgene $y w ; U A S-N a C h B a c 1$ and $y w ; U A S-N a C h B a c 4$ (gifts from Benjamin White, National Institute of Mental Health, Bethesda, MD) were used along with a control line carrying a nonconducting modified Drosophila open rectifier potassium (dORK) channel yw;UAS$d O R K \Delta-N C 1$, which were described previously (Nitabach et al., 2002, 2006). To express these channels in LNv neurons using the UAS-GAL4 system, which allows for targeted expression of a gene of interest (Brand and Perrimon, 1993), they were crossed with $y w ; p d f-G A L 4$ (Renn et al., 1999). For experiments to rescue rhythmic behavior in a $p d f^{01}$ background, $y w ; p d f-G A L 4 / U A S-P D F ; p d f^{01}$ and $y w ; p d f-G A L 4 / U A S-$ $N a C h B a c 4 ; p d f^{01}$ flies were generated using $y w ; U A S-P D F ; p d f^{01}$ and $y w$; $p d f-G A L 4 ; p d f^{01}$ (gifts from Paul Taghert, Washington University, St. Louis, $\mathrm{MO})$ and $y w$;UAS-NaChBac4. The line UAS-NaChBac4 was used for the $p d f^{01}$ experiments because $\mathrm{NaChBac}$ construct was inserted in chromosome 2 , allowing the creation of homozygous $p d f^{01}$ genetic background.

Behavioral analysis. TriKinetics Drosophila activity monitor system was used to assay the locomotor activity of individual flies. One- to four-day-old adults were placed singly in glass tubes and their activity was tracked by the breaking of an infrared beam. Activity counts were collected as a time series throughout the experiment in 15 min bins. For all assays, flies were subjected to at least $5 \mathrm{~d}$ of $12 / 12 \mathrm{~h} \mathrm{LD}$ cycles (light phase illumination was $50-100 \mathrm{~mW} / \mathrm{cm}^{2}$ ) followed by DD. Raw time series data obtained during DD conditions was analyzed using the Clocklab software from Actimetrics (Wilmette, IL) using the Lomb-Scargle method of time series analysis (Van Dongen et al., 1999). Peaks in the periodogram that extended above the $p=0.05$ cutoff line were considered to make a statistically significant contribution to the time series. The flies were categorized as arrhythmic, or exhibiting a single, double, or triple period based on the absence or presence of one, two or three peaks above the cutoff line. For the $p d f^{01}$ rescue experiments, the transgenic line UAS-NaChBac4 (referred to in the text as $\mathrm{NaChBac}$ ) was used and time series data from DD days 5-15 were analyzed and classified as described above. Negative control genotypes were $p d f$-GAL4 driver line and $U A S-N a C h B a c 4$ lines in a $p d f^{01}$ background. Positive controls were GAL4 driven UAS-PDF in $p d f^{01}$ background and UAS-NaChBac4 in a $p d f$ positive background. For all other experiments transgenic line UAS$\mathrm{NaChBacl}$ was used. The actograms of $y w ; p d f-G A L 4 ; N a C h B a c 1$ (referred to as $\mathrm{NaChBac}$ throughout the text) flies, which exhibited two simultaneous periods were visually examined on days 13-14 of DD to predict the timing of offset of activity on day 15 . Flies were sampled at four phases: $\mathrm{ON}$ (in the middle of the activity bout with shorter period $\sim 22 \mathrm{~h}$ ), OFF (in the middle of the activity bout with longer period $\sim 25$ h), $\mathrm{M}$ (the midpoint of the inactive phase after the short-period bout), and $\mathrm{M}^{*}$ (the midpoint of inactive phase after the long-period bout) for subsequent immunocytochemical analysis. For control flies exhibiting a single period, the offset of activity defined as circadian time 12 (CT12) were taken as the phase reference point and four phases were defined based on predicted offset of activity on day 15: CT1, CT6, CT11, and CT18, which were 11,6 , or $1 \mathrm{~h}$ before or $6 \mathrm{~h}$ after predicted offset of activity.

Electrophysiology. All patch-clamp measurements other than those explicitly indicated were made in the time interval between 6 and $12 \mathrm{~h}$ after "lights on" on flies maintained under $12 \mathrm{~h}$ LD cycles. For recordings in
DD, day 15 was chosen based on the recovery of behavioral rhythmicity in $\mathrm{NaChBac}$ flies, and the convergence of rest levels between $\mathrm{NaChBac}$ and control flies. Whole brains were dissected from adult flies in a dissection solution containing $20 \mathrm{U} / \mathrm{ml}$ papain (Worthington, Biochemicals, Lakewood, NJ) and $1 \mathrm{~mm}$ L-cysteine in standard external solution (composition given below). The brains were then placed in an RC-26 perfusion chamber (Warner Instruments, Hamden, CT) containing either standard or modified external solution to isolate $\mathrm{NaChBac}$ sodium channel (composition given below). Then the brain was stabilized using a holder described previously (Gu and O'Dowd, 2006). Green fluorescent protein (GFP)-positive lLNv were identified on the basis of their fluorescence using a BX51WI microscope (Olympus, Lehigh Valley, PA). A single recording was performed from one $1 \mathrm{LNv}$ per brain. All of the patch-clamp investigations were performed under continuous perfusion of the standard or modified external solutions. During the entire experiment the external solutions were aerated with a gas mixture of $95 \% \mathrm{O}_{2}$ and $5 \% \mathrm{CO}_{2}$. Composition of the standard external solution was (in $\mathrm{mM}$ ) $101 \mathrm{NaCl}, 1 \mathrm{CaCl}_{2}, 4 \mathrm{MgCl}_{2}, 3 \mathrm{KCl}, 5$ glucose, $1.25 \mathrm{NaH}_{2} \mathrm{PO}_{4}$ and 20.7 $\mathrm{NaHCO}_{3}, 250 \mathrm{mOsm}, \mathrm{pH}$ 7.2. The external solution modified to isolate $\mathrm{NaChBac}$ sodium currents contained (in $\mathrm{mm}$ ) $118 \mathrm{NaCl}, 2 \mathrm{CoCl}_{2}, 4$ $\mathrm{MgCl}_{2}, 3 \mathrm{KCl}, 5$ glucose, $10 \mathrm{HEPES}, 1$ tetraethylammonium, and 100 tetrodotoxin (TTX; to block endogenous TTX-sensitive sodium conductances), osmolality 250 mOsm, $\mathrm{pH}$ 7.2. $\mathrm{NaH}_{2} \mathrm{PO}_{4}$ and $\mathrm{NaHCO}_{3}$ were replaced in this solution to avoid precipitation of $\mathrm{CoCl}_{2} .10 \mathrm{M} \Omega$ resistance microelectrodes were fabricated from glass borosilicate capillaries by a PP-83 two-step gravity puller (Narishige, East Meadow, NY). Pipettes were filled with standard internal solution and directed onto the cells by an MP-285 micromanipulator (Sutter Instruments, Novato, CA). The composition of the standard internal solution was as follows (in $\mathrm{mm}$ ): $102 \mathrm{~K}$-gluconate, $0.085 \mathrm{CaCl}_{2}, 1.7 \mathrm{MgCl}_{2}, 17 \mathrm{NaCl}, 0.94 \mathrm{EGTA}$, and 8.5 HEPES, $235 \mathrm{mOsm}, \mathrm{pH}$ 7.2. The internal solution modified to isolate $\mathrm{NaChBac}$ sodium currents was $\mathrm{Cs}^{+}$-based containing (in $\mathrm{mM}$ ) 102 D-gluconic acid, $102 \mathrm{CsOH}, 0.085 \mathrm{CaCl}_{2}, 1.7 \mathrm{MgCl}, 17 \mathrm{NaCl}, 0.94$ EGTA, and 8.5 HEPES, $235 \mathrm{mOsm}, \mathrm{pH}$ 7.2. Two to three gigaohm seals were made in a cell-attached patch configuration and negative pressure was applied to break through into a whole-cell configuration. The liquid junction potential for this preparation was measured and found to be -5 $\mathrm{mV}$ and subtracted from all measurements of membrane potential. Voltage or current clamp recordings were using an Axopatch 200B amplifier (Molecular Devices, Palo Alto CA), digitized using Digidata 1322A acquisition system (Molecular Devices). Pulse protocols were controlled by the pClamp 8.2 Clampex software (Molecular Devices). In whole-cell configuration, whole-cell capacitance and series resistance compensations were applied. Recordings were low pass Bessel filtered at $5 \mathrm{kHz}$. Data analysis was performed by the pClamp 8.2 Clampfit software (Molecular Devices). Leak current was subtracted using the standard P/N leak subtraction method (that corrects for passive responses of the membrane) by applying a series $(\mathrm{N})$ number of sub-sweeps each $1 / \mathrm{Nth}$ of the amplitude of the main stimulus waveform applied.

Cell fills. Neurobiotin $(0.5 \%)$ was added to the internal recording electrode solution to perform cell fills. Pulses of depolarizing current of 30 $\mathrm{pA}$ were injected with a periodicity of $1.6 \mathrm{~s}$ for intervals ranging from 10 to $30 \mathrm{~min}$ to facilitate entry of neurobiotin into the neuronal projections.

Immunocytochemistry. Flies sampled at the four circadian time phases (as determined by their individual locomotor behavior) were removed from the behavioral monitoring apparatus, dissected, treated with collagenase, fixed with $4 \%$ paraformaldehyde at room temperature, rinsed with PBS with $1 \%$ Triton $\mathrm{X}-100$, and stored at $4^{\circ} \mathrm{C}$ until all of the brains of both genotypes from all phases were fixed. All of the samples were then subjected in parallel to the following steps: washes with PBS-1\% Triton $\mathrm{X}-100$, blocking with $10 \%$ horse serum, incubation with primary antibodies anti-PER (1:20,000; gift from R. Stanewsky, Queen Mary University of London, London, UK) and anti-PDF (1:3000; gift from J. Park, University of Tennessee, Nashville, TN); washes with PBS-0.5\% Triton X-100, incubation with anti-rabbit Alexa 488 and anti-rat Alexa 633 followed by more washes with PBS- $0.5 \%$ Triton X-100. Methodology for immunocytochemistry and antibody against PER and PDF are as described previously (Price et al., 1998; Nitabach et al., 2006). For cell-filled samples, a similar procedure was followed with Alexa-555 conjugated 
Streptavidin incubation for $24 \mathrm{~h}$ at $4^{\circ} \mathrm{C}$. Slides were mounted on slides using $50 \%$ glycerol with the ventral side facing upward.

Confocal imaging. Leica (Bannockburn, IL) TCS SP2 microscope with $63 \times$ objective was used to obtain images after the laser and PMT (photomultiplier tube) settings were standardized using the method described previously (Lin et al., 2004). Identical laser intensity, gain and offset settings were used for all samples. $1 \mu \mathrm{m}$ thick sections were obtained and maximum projection images of each neuronal subgroup were then analyzed using NIH image J software. The mean pixel intensity of each cell in a subgroup was measured individually and normalized using three replicate background values in the immediate vicinity of the cell and using the exact same area for sampling. Average pixel intensity values for each hemisphere from at least 8 brain hemispheres were used for further statistical analysis. The smoothed curves in the graphs for average staining intensity were generated using the analysis software from Origin (waterfall function), where the $\mathrm{ON}$ and $\mathrm{CT} 1$ data points have been plotted twice for continuity.

Statistical analysis. All statistical tests except time series analyses were done using Statistica. For behavioral assays, a $\chi^{2}$ test was performed to compare frequency distributions. For immunocytochemistry images, one-way ANOVA was performed for each cell group of each genotype with time point/phase as the independent variable and mean staining intensity of each brain hemisphere as dependent variable followed by post hoc multiple comparisons using Tukey's Honest Significant Difference Test.

\section{Results}

Altered electrical activity of LNv neurons causes initial arrhythmia followed by the emergence of split rhythmicity in activity/rest behavior

We showed previously that targeted expression of a low threshold voltage-gated sodium channel $\mathrm{NaChBac}$, in the LNv results in long-term stable concurrent multiple rhythms of locomotor activity with $\sim 60 \%$ of flies showing activity consolidated into at least two components in DD (Nitabach et al., 2006). Yet molecular oscillations show circuit-wide short-term desynchrony between the dorsal subgroups DN1 and DN2, compared with the sLNv, and LNd subgroup, when assayed on days 2 and 5 in DD (Nitabach et al., 2006) (supplemental Fig. 1, available at www. jneurosci.org as supplemental material). Furthermore, for such short-term desynchrony, we could not determine any association in the phases of PER or Par Domain Protein 1 (PDP1; an output molecule) cycling with the rhythmic phases of either of the two long-term bouts of activity. By comparison, controls expressing a nonconducting channel dORK-NC1 (Nitabach et al., 2002) show fairly synchronized oscillation among different neuronal subgroups for both PER and PDP1 protein expression (supplemental Fig. 1, available at www.jneurosci.org as supplemental material) (Lin et al., 2004). The anomaly between short-term desynchrony of molecular clock and long-term rhythmic behavior prompted us to examine the relationship between the electrophysiological state of the perturbed clock neurons and the molecular oscillations throughout the pacemaker circuit concurrent with the $\mathrm{NaChBac-induced} \mathrm{polyrhythmic} \mathrm{activity/rest} \mathrm{behavior.}$

First we verified that $\mathrm{NaChBac}$ expression in the $\mathrm{LNv}$ does not alter neuronal gross morphology (Fig. 1a) (compare Renn et al., 1999; Kaneko and Hall, 2000) In DD, activity rhythm of control flies free-run with a single period of $\sim 24 \mathrm{~h}$, whereas $\mathrm{NaChBac}$ flies show an initial spell of arrhythmicity for $\sim 5 \mathrm{~d}$ in DD after which their activity becomes organized into two distinct bouts; one exhibits a short $(\sim 22.5 \mathrm{~h})$ and the other long $(\sim 25 \mathrm{~h})$ period (Fig. 1b). To better evaluate this polyrhythmic locomotor behavior, the data were subjected to Lomb Scargle periodogram analysis (Van Dongen et al., 1999) by first analyzing the time series for the full length of the $14 \mathrm{~d}$ in DD followed by two separate analyses for days $1-5$ and days 5-14. In all three cases, the distribution of arrhythmic, single, and multiple periods in $\mathrm{NaChBac}$ flies was significantly different from controls $\left(\chi^{2}, p<0.001\right)$. Analysis of DD days 1-14 shows that whereas $>96 \%$ of control flies are rhythmic with a single period, $\sim 62 \%$ of $\mathrm{NaChBac}$ flies exhibit polyrhythmic locomotor behavior with two significant concurrently running periods (Fig. $1 c$, left). During days $1-5$, at least $86 \%$ of control flies exhibit a single period whereas the largest proportion of $\mathrm{NaChBac}$ flies are arrhythmic (65.4\%) (Fig. 1c, middle) and subsequently during days 5-14, a large proportion of flies exhibit two periods simultaneously (nearly 67\%) (Fig. 1c, right). Furthermore, there is no significant difference in the frequency distribution of rhythmic and arrhythmic flies between days $1-14$ and days $5-14$ in the NaChBac flies $\left(\chi^{2}=7.212, p=\right.$ $0.07)$. Thus, the double periodicity seen during days $1-14$ in DD is primarily accounted for by the activity during days $5-14$ and not by the activity during day $1-5$ (Fig. $1 c$ compare lower bars shaded blue). From visual examination of individual locomotor activity records (actograms), the most striking feature of arrhythmicity during the first $1-5 \mathrm{~d}$ is complete lack of organized activity and rest. We measured rest by calculating the total number of 15 min bins during which the flies were inactive. The average amount of rest in the control flies does not change over the course of $14 \mathrm{~d}$ in DD, indicating fairly stable levels of activity and rest (Fig. 1d). However, NaChBac flies show significantly lower rest initially, which increases progressively, and between days 10-14 becomes comparable with the controls (Fig. 1d). Most previous studies focused on sleep assays measure rest as $5 \mathrm{~min}$ intervals of inactivity, whereas we use longer intervals of $15 \mathrm{~min}$ bins as our threshold for assignment of rest [however, please refer to the use of $30 \mathrm{~min}$ bins for automated measurement of rest and the experimental justification therein for this bin length in the study by Hendricks et al. (2000)]. Therefore, it is likely that $\mathrm{NaChBac}$ flies rest as much as controls by days $10-14$, but whether such changes in rest throughout days 1-14 are caused by differential consolidation is unclear. The emergence of such a novel rhythmic behavioral pattern from initial arrhythmia and the convergence of rest levels between control and $\mathrm{NaChBac}$ flies after 2 weeks in DD led us to measure the electrophysiological properties and molecular oscillations on day 15 in DD.

\section{Electrophysiological properties of $\mathrm{LNv}$ neurons are altered by $\mathrm{NaChBac}$ expression and do not recover cell autonomously over time}

We measured the electrical activity of the lLNv neurons of control and $\mathrm{NaChBac}$-expressing flies in whole brains using whole-cell patch clamp (Gu and O'Dowd, 2006) at early (1- to 2-d-old flies maintained throughout their entire development in $12 \mathrm{~h} \mathrm{LD}$ ) and late (flies maintained in constant darkness, DD day 15) time points. ILNv neurons were identified using eGFP tag and recorded in whole cell current clamp or voltage clamp modes based on their better accessibility relative to sLNv neurons. Although immunocytochemical studies suggest that oscillations in PER, TIM or PDP-1 protein levels do not persist in ILNv in prolonged DD in contrast to the sLNv, our studies suggest that in fact membrane electrical properties continue to show circadian oscillation in DD in these neurons (Sheeba et al., 2008). Furthermore, mRNA levels of tim has been shown to exhibit robust oscillation in lLNv for up to $8 \mathrm{~d}$ in DD (Peng et al., 2003; Stoleru et al., 2005). Our preliminary attempts at recording from sLNv have yielded cell-attached measurements although we have not been able to reliably perform whole-cell measurements. These preliminary records suggest that sLNv show similar properties as $1 \mathrm{LNv}$ in 
a

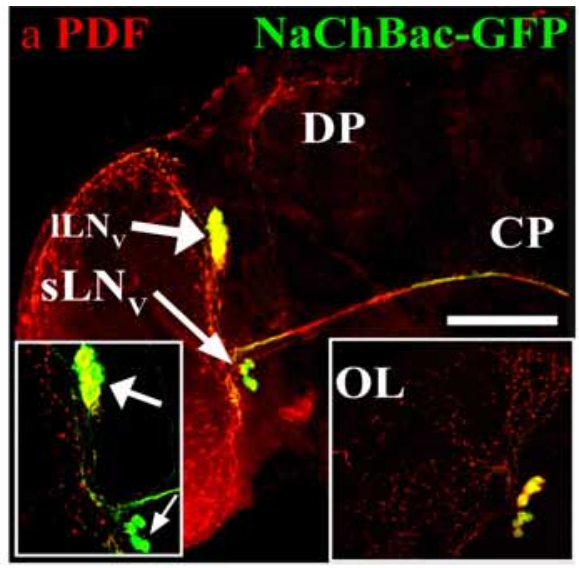

b

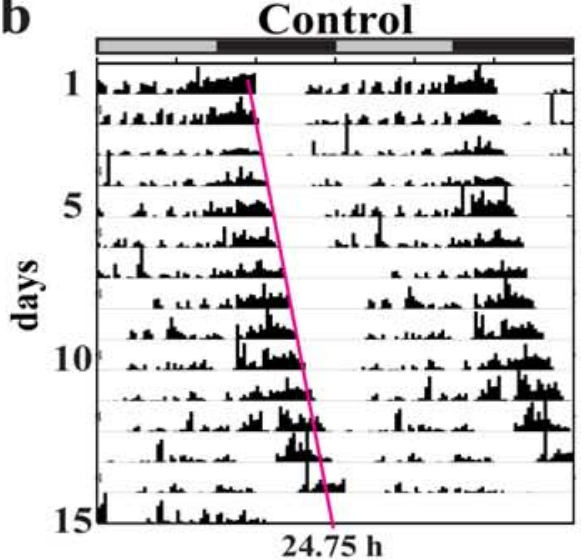

NaChBac

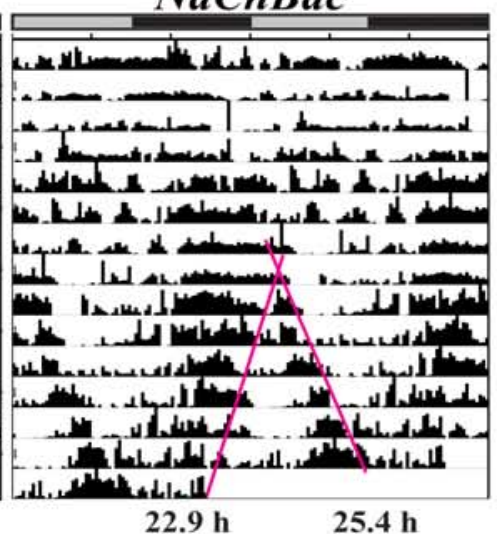

c

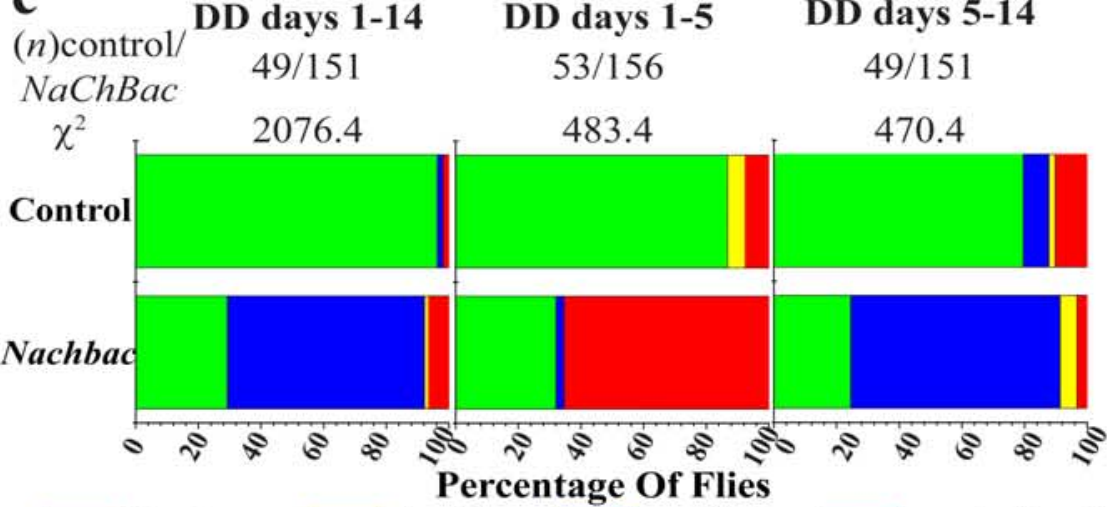

single $\square$ double $\square$ multiple $\square$ arrhythmic

d

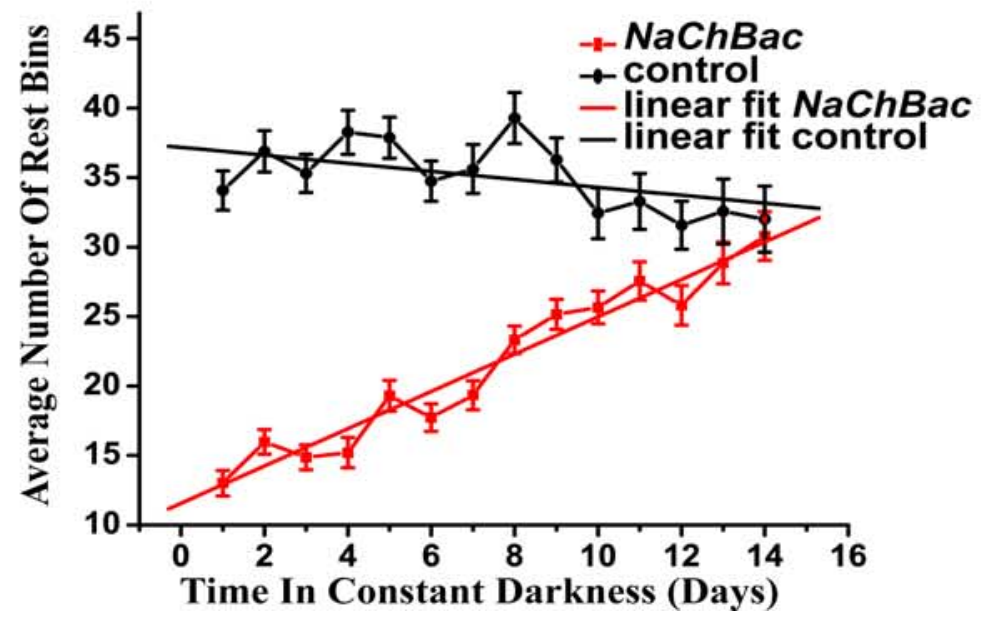

terms of oscillations in resting membrane potential, and firing frequency (data not shown). The identity of lLNv neurons was verified by cell fills using $0.5 \%$ neurobiotin followed by fluorescence-conjugated streptavidin (Fig. 2a1-a3). Inward currents are absent in whole-cell voltage clamp recordings of ILNv in $100 \mathrm{~nm}$ TTX-treated whole-brain preparations of LD 1- to 2-dold control flies (TTX blocks native Drosophila voltage-gated sodium currents) (Fig. 2b) whereas large voltage-dependent, low-threshold, slowly-inactivating TTXinsensitive inward sodium currents are seen in $\mathrm{NaChBac}$ lLNv recorded under the same conditions (Fig. 2c). NaChBac channels on ILNv neurons activate at $-65 \mathrm{mV}$ with largest current detected at $-55 \mathrm{mV}$ $(n=11)$ (Fig. $2 d)$, consistent with the biophysical properties of $\mathrm{NaChBac}$ cRNAinjected Xenopus oocytes (Ren et al., 2001; Nitabach et al., 2006).

In some cases, neurons are known to recover their normal intrinsic cell autonomous electrical properties and firing patterns over time after chronic perturbation (Turrigiano et al., 1994). To determine whether the gradual emergence of complex behavioral rhythmicity is caused by grad-

\footnotetext{
$\leftarrow$

Figure 1. Behavior of LNv-electrically altered flies is initially arrhythmic, which is then followed by emergence of complex rhythms. $\boldsymbol{a}$, Cell bodies and projections of GFPtagged $\mathrm{NaChBac-expressing} \mathrm{cells} \mathrm{(green)} \mathrm{immunostained}$ with anti-PDF antiserum (red). NaChBac is seen in cell bodies of sLNv (thin arrow) and ILNv (thick arrow) and in the processes of $s L N v$ in the dorsal protocerebral (DP), and contralateral projections (CP) of ILN . OL, Optic lobe. Scale bar, $80 \mu \mathrm{m}$. Inset, Higher magnification image of the LNv. $\boldsymbol{b}$, Examples of actograms of control ( $p d f-G A L 4 / U A S-d O R K-N C 1$ ) and $\mathrm{NaChBac}$ ( $p d f-G A L 4 / U A S-N a C h B a c$ ) flies in constant darkness. Gray and black bars above each actogram represent light and dark phases during previous entrainment in 12/12 $\mathrm{LD}$ cycle. Pink lines are aids to visualize free-running patterns and are not used for estimation of free-running period. c, As determined by Lomb-Scargle periodogram analysis, a significant proportion (62.9\%) of $\mathrm{NaChBac}$ flies exhibit two (blue) or more (yellow) periodicities during DD days 1-14 (left), whereas most (96.3\%) control flies exhibit a single period (green). For $\mathrm{NaChBac}$ flies, $65.5 \%$ were arrhythmic during days $1-5$ in $\mathrm{DD}$ (middle), whereas for this duration $>86 \%$ of controls show single rhythm. For days 5-14 in DD (right), $67 \%$ of $\mathrm{NaChBac}$ flies have two periods and only $24.5 \% \mathrm{ex}$ hibit single period, whereas $80 \%$ of controls show single period. The distribution of rhythm types is significantly different between control and $\mathrm{NaChBacflies} \mathrm{for} \mathrm{all} \mathrm{three} \mathrm{time} \mathrm{intervals.}$ $\left(\chi^{2}, p<0.001\right) . \boldsymbol{d}$, Average number of rest bins (left) and ratio of rest/normalized activity (right) on successive days in DD. Controls (black) do not show significant differences in rest levels, whereas $\mathrm{NaChBac}$ flies (red) start with significantly lower rest for the first $5 \mathrm{~d}$ in DD and then increase gradually. By day 14 , there is no significant difference either in average number of rest bins or ratios of rest/activity between control and $\mathrm{NaChBac}$ flies.
} 

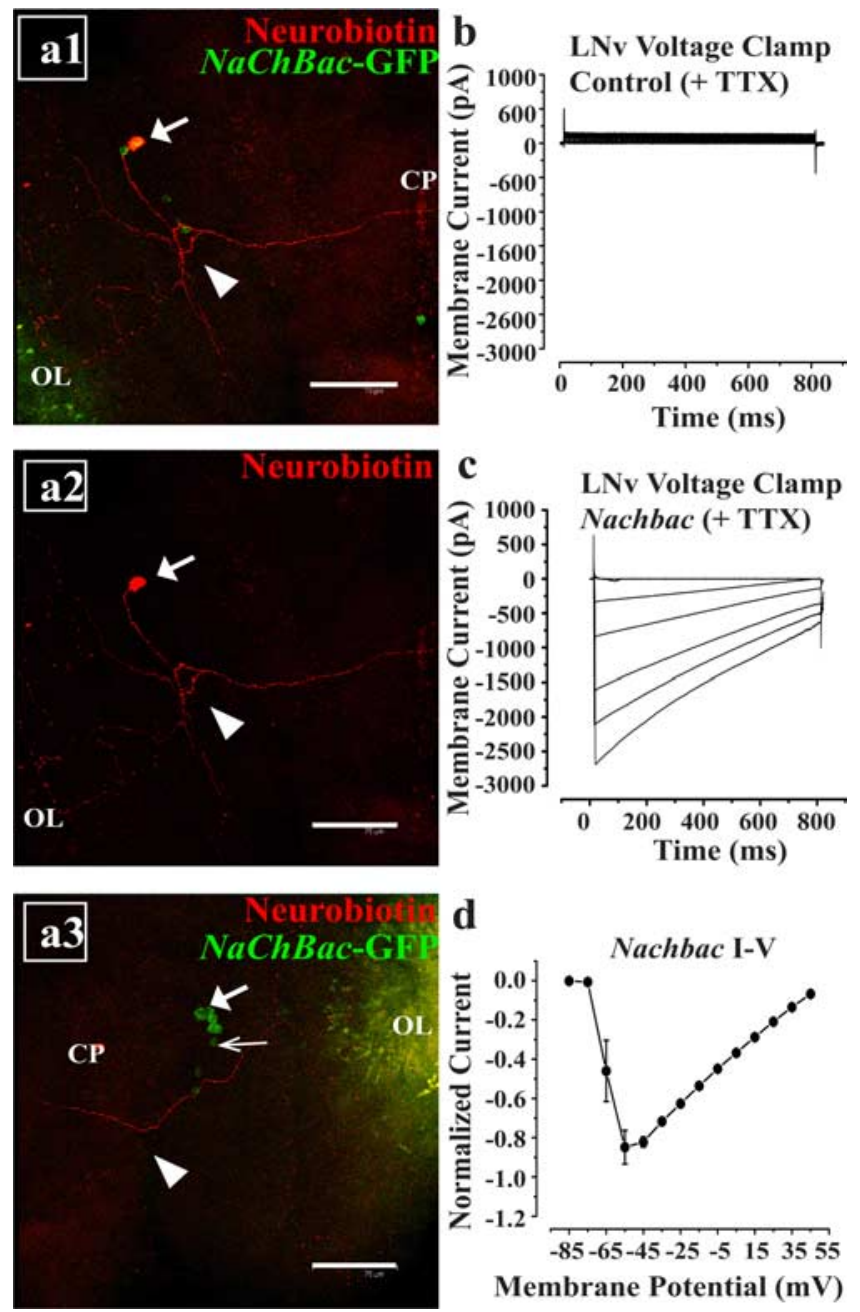

Figure 2. The electrophysiological properties of LNv neurons are altered by expression of voltage gated Sodium channel ( $\mathrm{NaChBac}$. a 1, a2, Neurobiotin-filled ILNv (thick arrow) labeled with fluorescence-conjugated Streptavidin colocalizes with GFP. a3, Contralateral projection innervating the opposite optic lobe (symbols and abbreviations are the same as in Fig. 1a). Scale bar, $75 \mu \mathrm{m}$. Expression of $\mathrm{NaChBac}$ results in TTX insensitive large voltage gated inward currents in ILNv. $\boldsymbol{b}, \boldsymbol{c}$, Whole-cell voltage-clamp records of TTX-treated ILNv of $(\boldsymbol{b})$ control and (c) $\mathrm{NaChBac}$ flies showing traces obtained at $20 \mathrm{mV}$ steps. $\mathrm{NaChBac}$ channels on ILNv neurons activate at $-65 \mathrm{mV}$ with largest current detected at $-55 \mathrm{mV}$. $\boldsymbol{d}$, Normalized current-voltage relationship of $\mathrm{NaChBac}$ flies. Mean and SEM values are indicated.

ual change in membrane properties of lLNv neurons after $\mathrm{NaCh}$ Bac expression, we recorded spontaneous action potentials and high-resolution continuous changes in membrane voltage using whole adult fly brain preparation on day 15 in DD, from control and $\mathrm{NaChBac}$-expressing lLNv neurons in current clamp mode. Spontaneous firing of control lLNv in the absence of holding current in whole-cell current-clamp mode is observed for up to $60 \min (n=10)$ (Fig. 3a1,c, black trace), with mean firing frequency of $2.3 \pm 0.4 \mathrm{~Hz}$ (mean $\pm \mathrm{SEM}$ ), mean action potential amplitude of $23.1 \pm 1.4 \mathrm{mV}$, and mean resting membrane potential of $-48 \pm 1 \mathrm{mV}$ (Table 1). Furthermore, in $\sim 30 \%$ of traces we see an oscillation in resting membrane potential with bursts of action potentials riding on crests of the oscillations (Fig. 3a2, inset shows trace detail). No significant differences were detectable in any of the measured electrophysiological parameters of ILNv of older flies that had been subjected to $15 \mathrm{~d}$ of DD (Fig. 3b). While whole-cell recording reveals detailed measurements of neuronal membrane voltage, this configuration creates a contin- uous solution between the neuronal cytoplasm and the recording pipette. Similar spontaneous firing rates of control lLNv is observed also in cell-attached recordings before whole-cell break in (data not shown), suggesting that these values are likely to reflect activity of healthy neurons. $\mathrm{NaChBac} \mathrm{LNv}$ show two patterns of high amplitude spontaneous depolarizations recorded in current clamp mode in the absence of holding current (Fig. 3c,d, Table 1). One of the NaChBac-induced firing patterns ( $n=8$ of 13 cells) (Fig. 3c) exhibits very large amplitude spontaneous depolarizations ( $144 \pm 5 \mathrm{mV}$ ) of short duration initiating from a hyperpolarized resting level $(-101 \pm 4 \mathrm{mV})$ with low frequency $(0.03 \pm$ $0.005 \mathrm{~Hz}$ ) relative to spontaneous action potential firing observed in control neurons. The other $\mathrm{NaChBac}$-induced firing pattern ( $n=7$ of 13 cells) (Fig. $3 d$ ) exhibits very large amplitude and long duration spontaneous depolarizations $(153 \pm 6 \mathrm{mV})$, also initiating from a hyperpolarized resting level $(-98 \pm 6 \mathrm{mV})$ with similar low frequency $(0.03 \pm 0.005 \mathrm{~Hz})$. Some NaChBacexpressing LNv neurons ( 2 of 13 cells) showed alternation between short- and the long-duration firing patterns (Fig. 3e). Both types of $\mathrm{NaChBac}$-induced spontaneous depolarizations initiate around $-100 \mathrm{mV}$, which is well below the $\mathrm{NaChBac}$ activation threshold as measured by voltage clamp in lLNv (Fig. $2 d$ ) or in Xenopus oocytes (Ren et al., 2001; Nitabach et al., 2006). This suggests that hyperpolarization-gated cation channels initiate these large spontaneous depolarization events.

No significant differences in depolarization pattern and amplitude or resting membrane potential are seen between membrane electrical properties of 1- to 2-d-old and 15-d-old control and $\mathrm{NaChBac}$ flies (Fig. 3a,b, compare gray traces, f,g, Table 1, phases LD ZT6 vs DD CT6 and equivalent phase comparison in $\mathrm{NaChBac}$ which is explained in detail below). This suggests that cell-autonomous homeostatic recovery to control intrinsic membrane electrical activity does not occur in $\mathrm{NaChBaclLNv}$ neurons even after long exposure to DD by which time robust complex behavioral rhythmicity emerges. Thus the emergence of behavioral rhythmicity out of initial arrhythmia occurs throughout the circuit rather than cell-autonomous recovery in the $\mathrm{NaChBac}$ expressing LNv neurons.

\section{Altered electrical activity in $\mathrm{LNv}$ neurons by $\mathrm{NaChBac}$ expression drives a single period bout of behavioral rhythmicity in the absence of PDF}

Many studies suggest that PDF synchronizes molecular clock cycling among different subgroups of clock neurons in Drosophila and is necessary for rhythmic expression of activity (HelfrichFörster et al., 2000; Park et al., 2000; Peng et al., 2003; Lin et al., 2004). At present, PDF is the only known circadian neurotransmitter released by the LNv neurons. We asked whether the effects of chronically altering electrical activity of LNv on behavioral activity rhythm are mediated via PDF by comparing the effects of $\mathrm{NaChBac}$ expression in LNv neurons in wild-type and $p d f^{01}$ null backgrounds. $\mathrm{NaChBac}$ expression and the absence of PDF in $p d f^{01}$ adult flies were verified by confocal microscopy by detection of GFP tagged NaChBac and anti-PDF antibody (Fig. 4a). $\mathrm{NaChBac}$ expression in LNv drives a robust single short-period behavioral rhythm in $p d f^{11}$ flies (Fig. $4 b$ third row, compare top and second rows, supplemental Fig. 2, available at www. jneurosci.org as supplemental material) as shown by the significantly larger proportion of flies with a single period compared with $p d f^{01}$ flies without $\mathrm{NaChBac}$ expression. This result suggests that the short-period locomotor rhythm is regulated by PDFindependent circadian signals. The degree of rhythmicity (percentage of rhythmic flies) in the $\mathrm{NaChBac}$ expressing $p d f^{01}$ flies is 
comparable with those in flies where PDF is reintroduced in LNv (Fig. 4b, compare third and fourth rows). The period of this single short-period rhythm is similar to that of a weak short-period rhythm seen in some $p d f^{11}$ flies in DD (supplemental Fig. 2, available at www.jneurosci.org as supplemental material) (Renn et al., 1999; Lin et al., 2004) and significantly longer than the short-period component of activity patterns of $p d f$ positive $\mathrm{NaCh}$ Bac flies in DD (Fig. 4b, compare third row mean of 22.9 h vs last row, $21.79 \mathrm{~h}$ ) ( $t$ test, $p<$ $0.05)$. Although the results of these studies cannot conclusively determine the origin of the short and long components of the rhythms in $\mathrm{NaChBac}$ flies in a $p d f$ positive background, we favor the hypothesis that in the absence of PDF the $\mathrm{NaChBac}$-induced long-period component of the complex rhythms is lost while the short-period component persists with a small alteration in period. Alternatively, the long- and shortperiod rhythms that arise in $\mathrm{NaChBac}$ flies in the presence of PDF may each be independently influenced by PDF. Although it is still unclear which neurons and neurotransmitters drive the shorter period component in the polyrhythmic NaChBac flies; the longperiod component is clearly PDF dependent.

\section{Prolonged exposure to DD causes} molecular oscillations throughout the pacemaker circuit to gradually reorganize into a novel pattern of polycyclic synchrony

Chronic altered excitability of the LNv neurons reorganizes acute and long-term circadian behavioral patterns (Fig. 1) (Nitabach et al., 2006). Because clock protein cycling is a marker of the circadian pacemaker circuit's functional state we asked whether gradual change into polyrhythmic locomotor behavior is a result of re-organization of the multiple neuronal subgroups that comprise the circadian pacemaker circuit. We determined previously that in the short-term, $\mathrm{NaChBac}$ expression in LNv results in circuit-wide desynchrony of molecular oscillation between the dorsal groups DN1 and 2, compared with the sLNv, and LNd when assayed after 2 or $5 \mathrm{~d}$ exposure to DD (Nitabach et al., 2006) (supplemental Fig. 1, available at www. jneurosci.org as supplemental material). These time points correspond to the initial behavioral arrhythmicity observed during the first $5 \mathrm{~d}$ in DD (Fig. 1b,c). Because stable rhythmic behavior emerges between 5 and $14 \mathrm{~d}$ in DD (Fig. 1b,c), we asked whether resynchronization of molecular oscillation occurs throughout the pacemaker circuit within this time interval. PER levels were semiquantitatively assayed by fluorescence immunocytochemistry in the PDF-
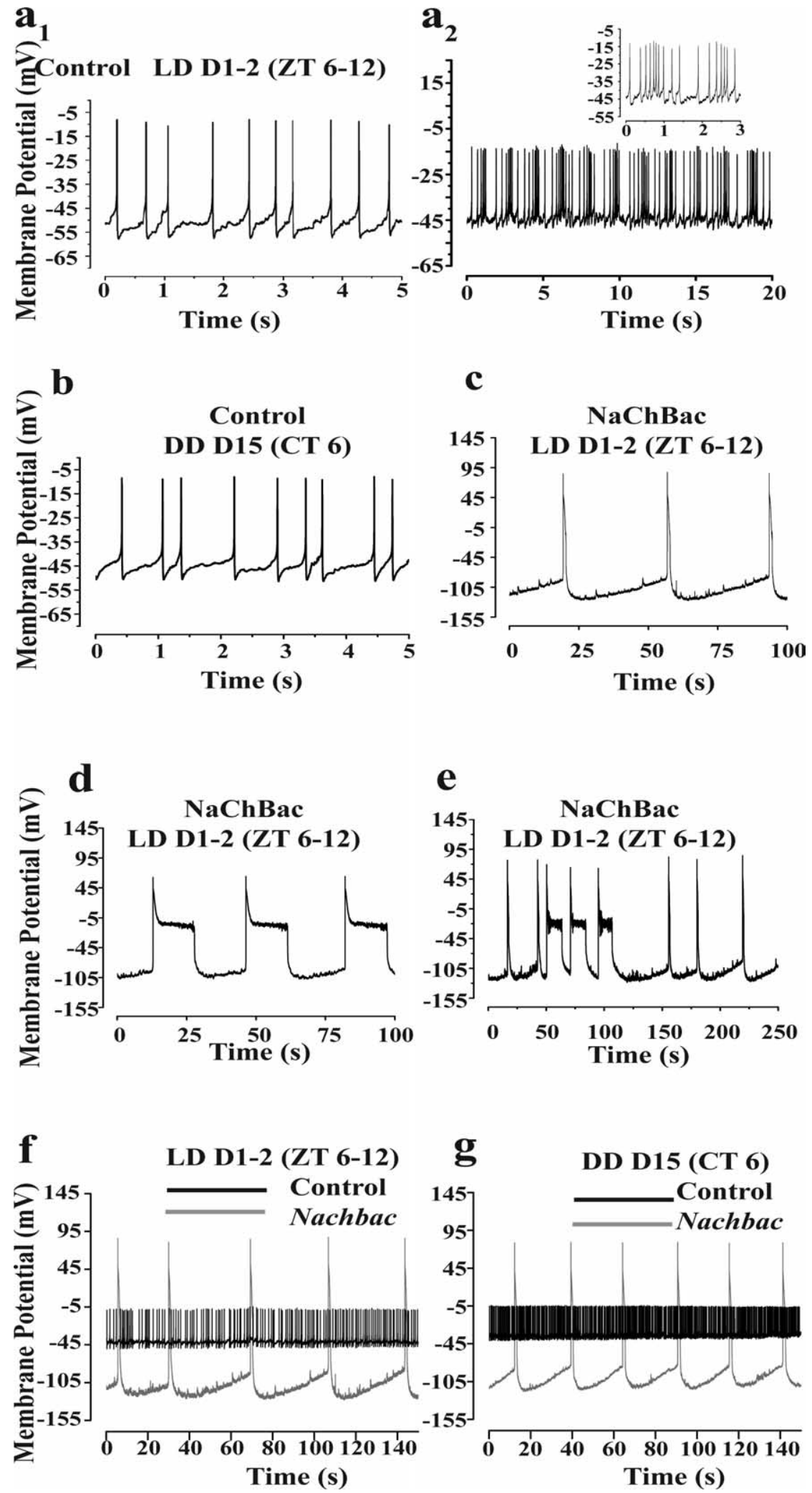

Figure 3. The intrinsic electrophysiological properties of electrically hyper-excited LNv neurons do not homeostatically recover to control values. $\boldsymbol{a}, \boldsymbol{b}$, Spontaneous action potentials of ILNv in control flies in LD day 1 Zeitgeber time (ZT) $6-12\left(\boldsymbol{a}_{\mathbf{1}}-\boldsymbol{a}_{\mathbf{2}}\right)$ and DD day 15 CT6 (b). Majority of cells show steady resting membrane potential of $\sim 48 \mathrm{mV}\left(a_{1}\right)$, whereas $\sim 30 \%$ showa small amplitude oscillation in membrane potential with bursts of action potentials riding on crests of membrane potential oscillations $\left(\boldsymbol{a}_{2}\right)$. Theinsetin $\boldsymbol{a}_{\mathbf{2}}$ shows detail of the action potential firing. $\boldsymbol{c}-\boldsymbol{e}, \mathrm{NaChBac}$ expression causes large depolarization of membrane potentials in ILNv. Two types of spontaneous depolarization patterns are seen.Somecellsshowlarge amplitude depolarization of short duration $(n=8 / 13)(c)$, whereas others showlong duration depolarization $(n=$ 7/13) with similar amplitude (d); furthermore, some cells tended to go back and forth between the two types of patterns (e). $\boldsymbol{f}, \boldsymbol{g}, \mathrm{NaChBac}$ expressing ILNv neurons do not recover to wild-type firing patterns after prolonged exposure to constant darkness. 
Table 1. The NaChBac expressing ILNv do not recover native physiological properties after prolonged exposure to DD

\begin{tabular}{|c|c|c|c|c|c|}
\hline & $\begin{array}{l}\text { Light condition } \\
\text { (time point) }\end{array}$ & $\begin{array}{l}\text { Firing } \\
\text { frequency }(\mathrm{Hz})\end{array}$ & $\begin{array}{l}\text { Action potential } \\
\text { amplitude }(\mathrm{mV})\end{array}$ & $\begin{array}{l}\text { Resting } \\
\text { membrane/plateau } \\
\text { potential (mV) }\end{array}$ & $n$ \\
\hline \multicolumn{6}{|l|}{ Control } \\
\hline LD (days 1-2) & ZT 6-12 & $2.28 \pm 0.41$ & $23.08 \pm 1.35$ & $-48 \pm 1$ & 10 \\
\hline DD (day 14) & CT 6 & $1.69 \pm 0.37$ & $25.48 \pm 3.90$ & $-46 \pm 2$ & 5 \\
\hline \multicolumn{6}{|l|}{$\mathrm{NaChBac}$} \\
\hline LD (days 1-2) & $\begin{array}{l}\text { ZT 6-12 (Fig. 3c,e) } \\
\text { ZT 6-12 (Fig. 3d,e) }\end{array}$ & $\begin{array}{l}0.03 \pm 0.005 \\
0.03 \pm 0.005\end{array}$ & $\begin{array}{l}144.3 \pm 5.09 \\
153.6 \pm 5.60\end{array}$ & $\begin{array}{l}-101 \pm 4 \\
-98 \pm 6 \text { (lower) } \\
-21 \pm 2 \text { (upper) }\end{array}$ & $\begin{array}{l}8 \\
7\end{array}$ \\
\hline DD (day 14) & M & $0.06 \pm 0.01$ & $146.66 \pm 5.99$ & $-105 \pm 6$ & 8 \\
\hline
\end{tabular}

Electrophysiological parameters (mean \pm SEM) are significantly different between control and $\mathrm{NaChBac}(p<0.0001)$. There are no significant differences in amplitude of action potential or resting membrane potential between LD (days 1-2) and DD (day 15) in control and NaChBac flies. The firing frequency of $\mathrm{NaChBac}$ flies is higher at DD day15 compared with LD (days $1-2 ; p<0.02$ ), but continues to be at least two orders of magnitude lower than controls; thus, it does not recover to control values. ZT, Zeitgeber time.

expressing LNv, as well as the LNd, DN1, DN2, and the PDFnegative fifth sLNv pacemaker neuronal subgroups, at four time points calibrated by the behavior of individual flies when complex rhythm is clearly evident at DD day 15 . Individual flies were removed from behavioral monitors under red light and immediately killed for immunocytochemistry. For controls (Fig. $5 a$ ) we chose four easily discernable phases using the offset of activity in DD as reference (CT12): 1, 6, and $11 \mathrm{~h}$ before offset, and $6 \mathrm{~h}$ after offset; denoted by CT11, CT6, CT1 and CT18, respectively, for PER estimation. For NaChBac flies (Fig. $5 a$ ) we chose four comparable time points designated as $\mathrm{ON}$ (during the activity bout corresponding to the shorter period), OFF (during the activity bout corresponding to the longer period), $\mathrm{M}$ (midpoint of rest interval after the ON bout and before the OFF bout), and $\mathrm{M}^{*}$ (midpoint of rest interval after the OFF bout and before the ON bout).

In controls, PER levels in different neuronal subgroups are inphase with each other with peaks at CT18 and/or CT1 and troughs at CT6 or CT11 (Fig. 5b, left, supplemental Fig. 3, available at www. jneurosci.org as supplemental material). We did not detect a statistically significant oscillation in PER in the ILNv of control or NaChBac flies (Fig. 5b). Unlike control flies, in NaChBac flies the PDFpositive sLNv, the LNd and the fifth sLNv are out of phase with each other. Furthermore, DN1 and DN2 neurons show two peaks of PER, one which is more or less in-phase with the LNd and the other that is in-phase with the PDF-positive sLNv. The two PER peaks in the DN1 and DN2 occur within the duration of a single PER cycle in the sLNv. Thus, in addition to multiple concurrent behavioral locomotor rhythms we also detect multiple molecular oscillator cycles distributed throughout the pacemaker circuit. The peak of PER in PDFnegative fifth sLNv occurs at comparable phases in control and $\mathrm{NaChBac}$ flies (Fig. 5b). In NaChBac flies the fifth sLNv peak is out-of-phase in comparison with the manipulated sLNv and the LNd. These results are consistent with those of some recent studies that indicate that the period and phase of molecular oscillation in the fifth sLNv is altered by environmental inputs independent of the other LNvs (Rieger et al., 2006; Bachleitner et al., 2007). However, the results of a recent study suggest that the fifth sLNv has similar projections as the other four sLNv and is therefore is likely to make intimate connections with the later (Helfrich-Förster et al., 2007). At the point of maximum divergence of the two free-running activity peaks in NaChBac flies, higher levels of PER protein in DN1 and DN2 groups coincide with higher levels of locomotor activity. Thus, peak PER levels in sLNv along with DN1 and DN2 is coincident with short-period activity bouts $(\sim 22.5 \mathrm{~h})$ whereas the peak in $\mathrm{LNd}$ is coincident with long-period activity bouts $(\sim 25 \mathrm{~h})$ in conjunction with DN1 and DN2. It is likely that the two peaks of DN1 is a reflection of the heterogeneity among DN1 (Helfrich-Förster et al., 2007). We also detect two peaks in PER levels in the DN2 cells of $\mathrm{NaChBac}$ flies, occurring during the two active phases, whereas controls show only one peak which is in phase with $\mathrm{DN} 1$ peak. This could be a reflection of an autonomous oscillation in DN2 occurring in addition to strong rhythmic inputs from $\mathrm{LNv}$ as a result of $\mathrm{NaChBac}$ expression. An association between the long-period bout and peak in PER levels in sLNv and short-period bout and fifth sLNv and one of the LNd was observed in another study using $c r y^{b}$ mutants, which also exhibit two bouts of activity each with a different period under constant light (LL) (Rieger et al., 2006). Based on these associations, we expect that the phase relationship of molecular oscillation among different neuronal subgroups will be internally consistent if measured at time points other than $15 \mathrm{~d}$ in $\mathrm{DD}$, with the exception of the special circumstance when long and short behavior bouts converge. At such time points, we expect an alignment of PER cycling between the fifth sLNv and LNv neuronal subgroups.

In contrast to the resynchrony of PER cycling among the neuronal subgroups, the membrane electrical properties of $1 \mathrm{LNv}$ do not recover (Table 1), suggesting that plasticity is distributed throughout the subgroups of neurons in the circadian pacemaker circuit rather than occurring cell-autonomously in the LNv neurons. Electrophysiological measurements of $\mathrm{NaChBac} \mathrm{LNv}$ across multiple time points under long-term DD conditions reveal the persistence of circadian oscillations in membrane physiological properties similar to controls (data not shown). Thus, $\mathrm{NaChBac}$ expression, although altering intrinsic membrane electrical properties, does not obliterate its rhythmic feature. Our results suggests that although different components of the circadian pacemaker circuit may regulate different aspects of activity behavior, they are coupled by strong interactions and that distribution of oscillators in the network is subject to plasticity evoked by changes in electrical activity or environmental conditions (Quintero et al., 2003; Schaap et al., 2003; Yamaguchi et al., 2003).

\section{Discussion}

The period values of the short and long-period activity bouts ( $\sim 22.5$ and $\sim 25 \mathrm{~h}$ ) observed in our studies closely match those reported earlier for several clock mutants of Drosophila (Helfrich and Engelmann, 1983; Helfrich, 1986; Yoshii et al., 2004; Nitabach et al., 2006; Rieger et al., 2006). The fact that robust multiple behavioral rhythms are observed in numerous mutant backgrounds strongly suggests that homeostatic behavioral resynchrony to period values of $\sim 22.5$ and $\sim 25 \mathrm{~h}$ is a circuit-level constraint rather than a property of any single subgroup of clock neurons. Nevertheless, we note that the two periods that emerge in the mutant $c r y^{b}$ under dim LL undergo continuous and opposite changes in period length with increasing light intensity (Yoshii et al., 2004). Thus, it is likely that inputs perceived by individual components of the pacemaker circuit may differentially influence components of the circuit during the intermediary or transition states, whereas the steady state period is determined by the entire network. Although many studies have proposed the dominance of the sLNv in determining the freerunning rhythm in DD (Helfrich-Förster, 1998; Blanchardon et 
a

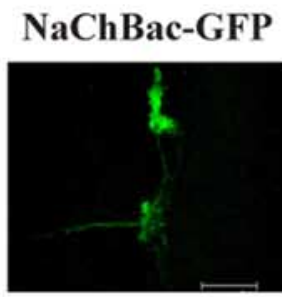

$\alpha$ PDF
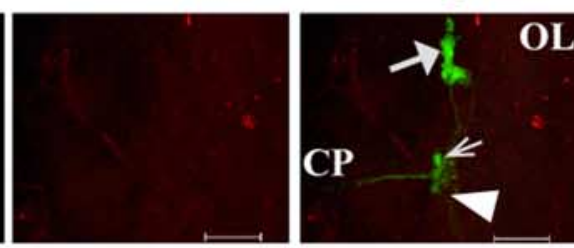

yw;pdfGAL4/UAS NaChBac4;pdf ${ }^{11}$

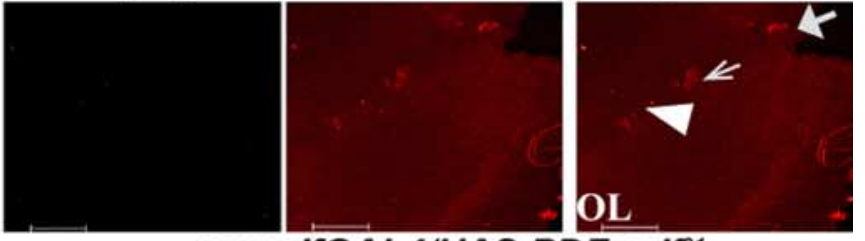

yw;pdfGAL4/UAS PDF;pdfo1

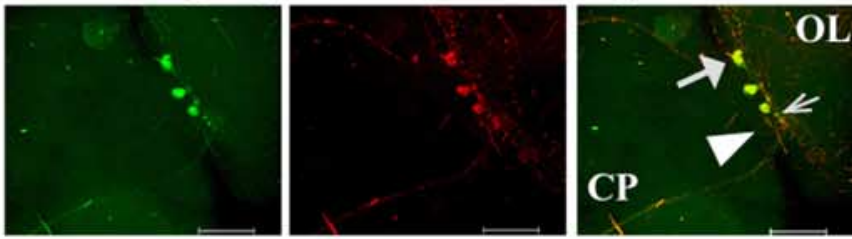

yw;pdfGAL4/UAS NaChBac4;+

b

Genotype

pdfor
pdw;
pdfGAL4;
pdf

yw;pdfGAL4/
UAS NaChBac4;
pdf

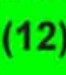

Period(h)

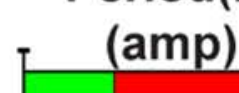

$22.40 \pm 0.15$

(12)
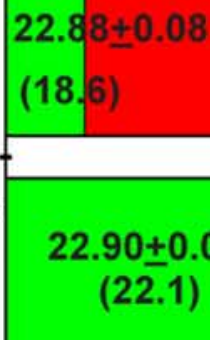

$22.90 \pm 0.08$

(22.1)

$\chi 2 \mathbf{N}$

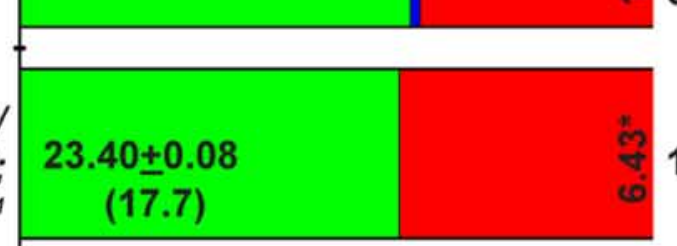

68

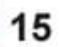

UAS PDF;

pdfor

(17.7)

yw;pdfGAL4/

UAS NaChBac4;
$24.62 \pm 0.15$

$21.79 \pm 0.09^{\mathrm{a}}(21.5)$ $(30.3)$ $24.95 \pm 0.09^{\mathrm{b}}(35.7)$
$+$

$2 \vee$ al., 2001; Grima et al., 2004), previous reports suggest that the molecular oscillations in DN1 neurons play an important role in determining rhythmic activity/rest behavior both under DD and LL conditions (de la Paz Fernandez et al., 2007; Murad et al., 2007). The results of a study which manipulated molecular oscillations in circadian pacemaker circuit by targeted expression of SHAGGY (SGG; a clock component whose overexpression speeds up the oscillations in mRNA of circadian genes) (Stoleru et al., 2005) suggests that whereas sLNv, LNd, DN1, and DN3 cells are part of a circuit that regulates locomotor activity rhythm, the $1 \mathrm{LNv}$ and DN2 cells form a separate and independent circuit that apparently does not influence locomotor activity rhythm and that the DN2 cells are the dominant component of this second circuit and regulate the oscillations in the ILNv (Stoleru et al., 2005).

Is there a clear association with the phase of molecular oscillation in any of the circadian pacemaker neurons with the phase of behavioral locomotor activity? Under LD, wild-type flies show two peaks in locomotor activity, yet, all known pacemakers exhibit a synchronized phase of PER oscillation that peaks coincident with the morning peak in activity. Wild-type flies when subjected to DD show a single peak in activity (apparently a derivative of the evening peak), and the peak in PER levels of all cells remain synchronized for the first few days, occurring at the trough of behavioral activity. After $\sim 5 \mathrm{~d}$ in DD, the phase of peak PER levels appears to drift apart among the members of the circuit, with DN2 cells being out-of-phase, as determined by sampling at $12 \mathrm{~h}$ (Veleri et

\section{$\leftarrow$}

Figure 4. LNv electrical hyperexcitation rescues rhythmicity in adult $p d f^{01}$ flies. $a$, Anti-PDF confirms the absence of PDF in yw;pdf-GAL4/UAS-NaChBac4;pdf ${ }^{01}$ flies. NaChBac expression in LNv is confirmed by GFP (symbols and abbreviations are the same as in Fig. 1a). Scale bar, $75 \mu \mathrm{m}$. $\boldsymbol{b}$, Frequency distribution of activity rhythm phenotypes: arrhythmic (red), single period (green), and double period rhythm (blue) assayed during days 5-15 in DD. Control flies with either UAS-NaChBac transgene (top row) or pdf-GAL4 driver (second row) are predominantly arrhythmic in a $p d f^{01}$ background. $A \chi^{2}$ test shows a significantly larger fraction of rhythmic flies compared with controls for both yw;pdf-GAL4/ UAS-NaChBac4;pdf (third row) and yw;pdf-GAL4/UAS-PDF; $p d f^{01}$ (fourth row). yw;pdf-GAL4/UAS-NaChBac4; + flies show predominantly double period rhythm (bottom row). The mean ( \pm SEM) of the free-running period of single and double period rhythms are indicated within the green- and blue-labeled regions, with mean amplitude of the rhythm in parentheses. $\boldsymbol{a}$, Mean short-period rhythm; $\boldsymbol{b}$, mean longperiod rhythm. ${ }^{*} p<0.02$; ${ }^{* *} p<0.001$. n.s., No significant difference. 
a
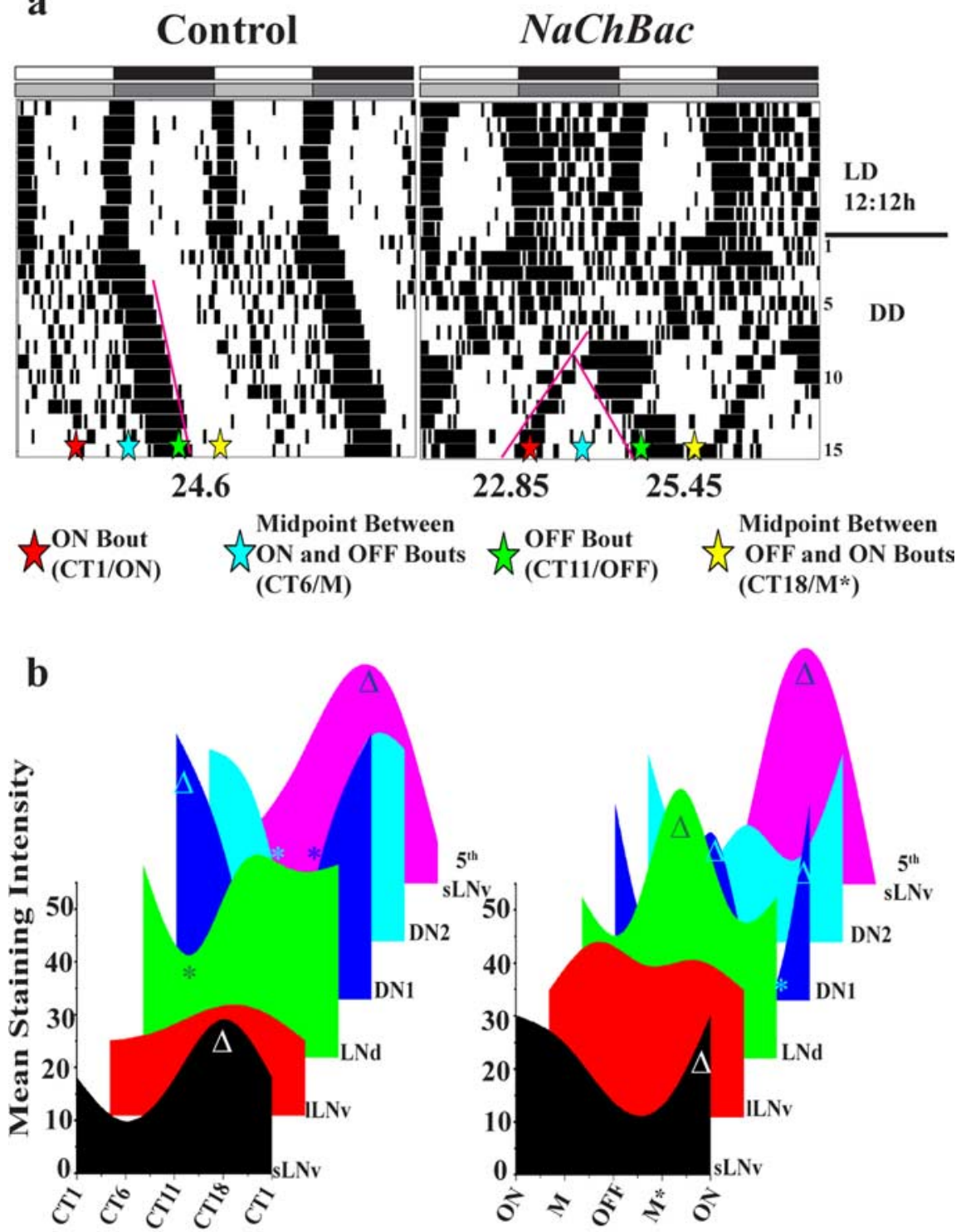

Time Points For Dissection

Figure 5. Long-term homeostatic resynchronization of behavioral rhythms and molecular oscillation in flies with electrically hyper-excited LNv. $\boldsymbol{a}$, Representative actograms of control and $\mathrm{NaChBac}$ flies depicting time points at which flies were removed from behavioral monitors and sampled for anti-PER immunocytochemistry. Control flies were killed at CT1, CT6, CT11, and CT18 and $\mathrm{NaChBac}$ flies were dissected at $\mathrm{ON}, \mathrm{OFF}, \mathrm{M}$, and $\mathrm{M}^{*}$ phases (for details, see Results). Bars above actograms denote day (white) and night (black) for first $11 \mathrm{~d}$ when flies were subjected to a $12 \mathrm{~h} \mathrm{LD}$ cycle before release into DD. The pink lines show free-running patterns, stars denote time of dissection. $\boldsymbol{b}$, Mean staining intensity is plotted against phase of sampling for subgroups sLNv, ILNv, LNd, DN1, DN2, fifth sLNv. ${ }^{\Delta}$ Statistically significant peaks; ${ }^{*} p<0.05$, statistically significant troughs.

corresponding to the peak activity of each of the two polyrhythmic bouts in LL in $c r y^{b}$ mutants (Rieger et al., 2006) and a subset of LNd appears to be in phase with the PDF negative fifth sLNv and has high PER levels during one of two activity peaks (the shortperiod bout apparently derived from the LD morning peak). For flies expressing $\mathrm{NaChBac}$ in the LNv, the phase distribution of PER cycling between the different pacemaker neuronal subgroups shows an antiphasic relationship between the PDFpositive and PDF-negative subsets of sLNv, but is opposite to that seen in $\mathrm{cry}^{b}$ flies under LL (sampled at $\sim 6 \mathrm{~h}$ intervals). Furthermore, we see two peaks in PER levels in DN1 and DN2 and also a clear oscillation in LNd that is not seen in controls or any other study in prolonged DD. Thus, the association between the short-period and long-period activity bouts with the PDF positive and negative $s L N v$ is labile and is highly dependent on environmental or intercellular signals. Furthermore, the polyrhythmicity in behavior and novel pattern of synchrony among the different members of the circadian pacemaker circuit in $\mathrm{NaChBac}$ flies appears to be a result of the alteration of electrophysiological properties in either the sLNv, $1 \mathrm{LNv}$, or both types of LNv.

To summarize what has been observed for the temporal relationship between clock cycling in the pacemaker neurons and overt locomotor behavior, from our results and those from previous studies, we conclude that there is no absolute relationship between activity level and the phase of PER cycling in sLNv. This is particularly clear when comparing the experimental conditions which lead to two behavioral activity peaks, including the morning and evening bouts for wild type flies in LD; and polyrhythmic bouts seen for LL-cry ${ }^{b}$; DD$\mathrm{NaChBac}$ expressed in the LNv. Furthermore, we propose that the relationship between the pattern of molecular oscillations among the different neuronal subgroups and rhythmic activity rest behavior in the

al., 2003) or $6 \mathrm{~h}$ intervals (Nitabach et al., 2006). When PER is overexpressed in the pacemaker circuit using the tim-GAL4 driver, it is possible to induce a robust single-period rhythm under LL (Murad et al., 2007) in $>90 \%$ of flies. In this case, molecular oscillation appears to persist in DN1 up to the fourth day in LL as quantified by cell counts based on PDP-1 staining (Murad et al., 2007). The peak in PDP-1-immunopositive cell numbers coincides with falling levels of locomotor activity (although not the trough) (Murad et al., 2007). Two simultaneously occurring behavioral rhythms are seen in LL in $c r y^{b}$ mutants, with increasing fraction of flies showing polyrhythmic locomotor behavior with increasing light intensity (Yoshii et al., 2004). PER levels in the PDF positive sLNv and the PDF negative fifth sLNv are antiphasic as determined by sampling at time points ( $12 \mathrm{~h}$ intervals) absence of external time cues under DD and LL is likely to be a result of continuous recalibration of signals being perceived by members of the pacemaker circuit. When these signals are below a certain threshold the circuit remains fairly tightly synchronized in terms of the molecular oscillation of clock proteins. Whereas when membrane electrical properties of parts of the circuit are altered to produce higher-than-threshold signals, molecular oscillations in the circuit are rendered asynchronous along with a loss of temporally regulated behavioral activity.

We show that the circuit gradually recovers from perturbations applied to parts of the network via circuit-wide plasticity as demonstrated by the emergence of novel behavior pattern with novel consolidated activity patterns and synchrony in molecular oscillation in different subgroups of the circuit. The electrically 
perturbed LNv do not recover their normal pattern of spontaneous action potential firing. Previous work shows that current injection evokes firing in otherwise silent large LNv (Park and Griffith, 2006). The absence of spontaneous firing in this previous study is probably caused by technical differences in the recording procedures (compare Park and Griffith, 2006; Gu and O'Dowd, 2006). The results of our study provide empirical evidence for the idea first proposed by Pittendrigh and Daan (1976) that gradual resynchrony among a large number of constituent oscillators could account for initial arrhythmicity and subsequent emergence of rhythmic behavior seen in animals when exposed to LL. More recent studies in mammals indicate a mechanism for plasticity in neural networks and outputs involving emergence of a coherent oscillation from previously asynchronous oscillator cells in sub areas of SCN when animals are transferred to DD from LL (Yan et al., 2005). In summary, the adult circadian pacemaker circuit exhibits electrical-activity dependent circuit-level plasticity of oscillators as shown by the reorganization of molecular oscillation and rhythmic activity/rest behavior. Electrophysiological studies of pacemaker cells in the accessory medulla of cockroaches has led to the hypothesis that pacemaker cells are organized into assemblies with distinct phases which are synchronized by neurotransmitters such as PDF and GABA (Schneider and Stengl, 2005). In Drosophila we show clear evidence for the existence of PDF-independent factors that contribute toward the regulation of circadian activity/rest rhythm also suggesting the existence of compensatory/redundant mechanisms in the pacemaker neuronal circuit. We hypothesize that the emergence of rhythmic activity due to $\mathrm{NaChBac}$ expression using $p d f G A L 4$ driver in $p d f^{01}$ flies may cause the rhythmic release of yet unknown neurotransmitters that are otherwise released at lower levels than would be necessary to elicit robust rhythmic behavior. Electrophysiological measurements of $\mathrm{NaChBac}$ expressing flies have shown that ILNv show giant action potentials, which may likely trigger downstream neurons to release, signals that can compensate for the lack of PDF. Together, the results of our experiments along with those of others that have examined the relationship between activity and the pattern of phase distribution among the different pacemaker components (Veleri et al., 2003; Yoshii et al., 2004; Nitabach et al., 2006; Rieger et al., 2006; Miyasako et al., 2007) suggests that different genetic manipulations and environmental conditions place the architecture of the pacemaker circuit to unique steady states, each of which is able to use different components of the circadian pacemaker circuit to generate distinct circadian patterns in behavior.

\section{References}

Bachleitner W, Kempinger L, Wulbeck C, Rieger D, Helfrich-Förster C (2007) Moonlight shifts the endogenous clock of Drosophila melanogaster. Proc Natl Acad Sci USA 104:3538-3543.

Blanchardon E, Grima B, Klarsfeld A, Chelot E, Hardin PE, Preat T, Rouyer F (2001) Defining the role of Drosophila lateral neurons in the control of circadian rhythms in motor activity and eclosion by targeted genetic ablation and PERIOD protein overexpression. Eur J Neurosci 13:871-888.

Brand AH, Perrimon N (1993) Targeted gene expression as a means of altering cell fates and generating dominant phenotypes. Development 118 : 401-415.

de la Paz Fernandez M, Chu J, Villella A, Atkinson N, Kay SA, Ceriani MF (2007) Impaired clock output by altered connectivity in the circadian network. Proc Natl Acad Sci USA 104:5650-5655.

Grima B, Chelot E, Xia R, Rouyer F (2004) Morning and evening peaks of activity rely on different clock neurons of the Drosophila brain. Nature 431:869-873

Gu H, O’Dowd DK (2006) Cholinergic synaptic transmission in adult Drosophila Kenyon cells in situ. J Neurosci 26:265-272.
Helfrich C (1986) Role of the optic lobes in the regulation of the locomotor activity rhythm of Drosophila melanogaster: behavioral analysis of neural mutants. J Neurogenetics 3:321-343.

Helfrich C, Engelmann W (1983) Circadian rhythms of locomotor activity in Drosophila melanogaster and its mutants sine occulis and small optic lobes. Physiol Entomol 8:257-272.

Helfrich-Förster C (1998) Robust circadian rhythmicity of Drosophila melanogaster requires the presence of lateral neurons: a brain-behavioral study of disconnected mutants. J Comp Physiol A Neuroethol Sens Neural Behav Physiol 182:435-453.

Helfrich-Förster C, Tauber M, Park JH, Muhlig-Versen M, Schneuwly S, Hofbauer A (2000) Ectopic expression of the neuropeptide pigmentdispersing factor alters behavioral rhythms in Drosophila melanogaster. J Neurosci 20:3339-3353.

Helfrich-Förster C, Shafer OT, Wulbeck C, Grieshaber E, Rieger D, Taghert P (2007) Development and morphology of the clock-gene-expressing lateral neurons of Drosophila melanogaster. J Comp Neurol 500:47-70.

Hendricks JC, Finn SM, Panckeri KA, Chavkin J, Williams JA, Sehgal A, Pack AI (2000) Rest in Drosophila is a sleep-like state. Neuron 25:129-138.

Kaneko M, Hall JC (2000) Neuroanatomy of cells expressing clock genes in Drosophila: transgenic manipulation of the period and timeless genes to mark the perikarya of circadian pacemaker neurons and their projections. J Comp Neurol 422:66-94.

Kaneko M, Helfrich-Förster C, Hall JC (1997) Spatial and temporal expression of the period and timeless genes in the developing nervous system of Drosophila: newly identified pacemaker candidates and novel features of clock gene product cycling. J Neurosci 17:6745-6760.

Lin Y, Stormo GD, Taghert PH (2004) The neuropeptide pigmentdispersing factor coordinates pacemaker interactions in the Drosophila circadian system. J Neurosci 24:7951-7957.

Miyasako Y, Umezaki Y, Tomioka K (2007) Separate sets of cerebral clock neurons are responsible for light and temperature entrainment of Drosophila circadian locomotor rhythms. J Biol Rhythms 22:115-126.

Murad A, Emery-Le M, Emery P (2007) A subset of dorsal neurons modulates circadian behavior and light responses in Drosophila. Neuron 53:689-701.

Nitabach MN, Blau J, Holmes TC (2002) Electrical silencing of Drosophila pacemaker neurons stops the free-running circadian clock. Cell 109:485-495.

Nitabach MN, Sheeba V, Vera DA, Blau J, Holmes TC (2005) Membrane electrical excitability is necessary for the free-running larval Drosophila circadian clock. J Neurobiol 62:1-13.

Nitabach MN, Wu Y, Sheeba V, Lemon WC, Strumbos J, Zelensky PK, White $\mathrm{BH}$, Holmes TC (2006) Electrical hyperexcitation of lateral ventral pacemaker neurons desynchronizes downstream circadian oscillators in the fly circadian circuit and induces multiple behavioral periods. J Neurosci 26:479-489.

Park D, Griffith LC (2006) Electrophysiological and anatomical characterization of PDF-positive clock neurons in the intact adult Drosophila brain. J Neurophysiol 95:3955-3960.

Park JH, Helfrich-Förster C, Lee G, Liu L, Rosbash M, Hall JC (2000) Differential regulation of circadian pacemaker output by separate clock genes in Drosophila. Proc Natl Acad Sci USA 97:3608-3613.

Peng Y, Stoleru D, Levine JD, Hall JC, Rosbash M (2003) Drosophila freerunning rhythms require intercellular communication. PLoS Biol 1:E13.

Petri B, Stengl M (1997) Pigment-dispersing hormone shifts the phase of the circadian pacemaker of the cockroach Leucophaea maderae. J Neurosci 17:4087-4093.

Pittendrigh C, Daan S (1976) A functional analysis of circadian pacemakers in nocturnal rodents. V. Pacemaker complexity: a clock for all seasons. J Comp Physiol 106:333-335.

Price JL, Blau J, Rothenfluh A, Abodeely M, Kloss B, Young MW (1998) double-time is a novel Drosophila clock gene that regulates PERIOD protein accumulation. Cell 94:83-95.

Quintero JE, Kuhlman SJ, McMahon DG (2003) The biological clock nucleus: a multiphasic oscillator network regulated by light. J Neurosci 23:8070-8076.

Ren D, Navarro B, Xu H, Yue L, Shi Q, Clapham DE (2001) A prokaryotic voltage-gated sodium channel. Science 294:2372-2375.

Renn SC, Park JH, Rosbash M, Hall JC, Taghert PH (1999) A pdf neuropeptide gene mutation and ablation of PDF neurons each cause severe abnormalities of behavioral circadian rhythms in Drosophila. Cell 99:791-802. 
Rieger D, Shafer OT, Tomioka K, Helfrich-Förster C (2006) Functional analysis of circadian pacemaker neurons in Drosophila melanogaster. J Neurosci 26:2531-2543.

Schaap J, Albus H, VanderLeest HT, Eilers PH, Detari L, Meijer JH (2003) Heterogeneity of rhythmic suprachiasmatic nucleus neurons: implications for circadian waveform and photoperiodic encoding. Proc Natl Acad Sci USA 100:15994-15999.

Schneider NL, Stengl M (2005) Pigment-dispersing factor and GABA synchronize cells of the isolated circadian clock of the cockroach Leucophaea maderae. J Neurosci 25:5138-5147.

Sheeba V, Gu H, Sharma VK, O’Dowd DK, Holmes TC (2008) Circadianand light-dependent regulation of resting membrane potential and spontaneous action potential firing of Drosophila circadian pacemaker neurons. J Neurophysiol, in press.

Stoleru D, Peng Y, Nawathean P, Rosbash M (2005) A resetting signal between Drosophila pacemakers synchronizes morning and evening activity. Nature 438:238-242.

Turrigiano G, Abbott LF, Marder E (1994) Activity-dependent changes in the intrinsic properties of cultured neurons. Science 264:974-977.
Van Dongen HP, Olofsen E, VanHartevelt JH, Kruyt EW (1999) A procedure of multiple period searching in unequally spaced time-series with the Lomb-Scargle method. Biol Rhythm Res 30:149-177.

Veleri S, Brandes C, Helfrich-Förster C, Hall JC, Stanewsky R (2003) A selfsustaining, light-entrainable circadian oscillator in the Drosophila brain. Curr Biol 13:1758-1767.

Yamaguchi S, Isejima H, Matsuo T, Okura R, Yagita K, Kobayashi M, Okamura H (2003) Synchronization of cellular clocks in the suprachiasmatic nucleus. Science 302:1408-1412.

Yan L, Foley NC, Bobula JM, Kriegsfeld LJ, Silver R (2005) Two antiphase oscillations occur in each suprachiasmatic nucleus of behaviorally split hamsters. J Neurosci 25:9017-9026.

Yoshii T, Funada Y, Ibuki-Ishibashi T, Matsumoto A, Tanimura T, Tomioka K (2004) Drosophila cry $^{b}$ mutation reveals two circadian clocks that drive locomotor rhythm and have different responsiveness to light. J Insect Physiol 50:479-488.

Yu W, Hardin PE (2006) Circadian oscillators of Drosophila and mammals. J Cell Sci 119:4793-4795. 